Original Article

\title{
The effectiveness of home-based exercise programs for low back pain patients
}

\author{
SEvgi ÖZdinç AnAR, PhD ${ }^{1)}$ \\ 1) Physical Therapy and Rehabilitation Department, Health Science Faculty, Trakya University: \\ Edirne 22030, Turkey
}

\begin{abstract}
Purpose] The purpose of this study was to investigate the effectiveness of home-based exercise programs for low back pain (LBP) patients. [Subjects and Methods] The study subjects were 49 volunteer chronic LBP patients. Home-based exercises that had been specifically modified for the individual patients were prescribed for a period of four weeks, and the volunteers were asked to return for a control evaluation at the end of this period. Exercise adherence and correct performance, pain intensity, disability, endurance, and flexibility were compared between pre- and post intervention, and correlations between exercise adherence and correct performance with, pain intensity score, endurance, disability, and flexibility were investigated. [Results] Twenty-eight patients (57.14\%) did not return for the control evaluation. The mean age of the patients who participated in this control test was 43.24 \pm 10.89 years. The adherence rate of the home exercise program was $54.10 \pm 26.01 \%$, and the correctly performed execises score was $2.7 \pm 1.9$. All of the parameters had improved at the final evaluation; however, there was no correlation among the parameters. [Conclusion] Clinicians should be aware of the patient's adherence level when recommending home-based exercises, and should also realize that exercises might be performed inaccurately in an unsupervised environment.

Key words: Low back pain, Exercise adherence, Exercise
\end{abstract}

(This article was submitted Apr. 11, 2016, and was accepted Jun. 9, 2016)

\section{INTRODUCTION}

Low back pain (LBP) is the most common musculoskeletal problem and the most common cause of disability in people aged $45-65^{1)}$. It is also a socio-economic problem since LBP is the most common cause of workforce absenteeism ${ }^{1,2)}$. It is classified as chronic when the complaints last longer than three months ${ }^{3,4)}$. Approximately $15-45 \%$ of the population are affected by chronic $\mathrm{LBP}^{1)}$.

Exercise is one of the rare evidence-based treatments for non-specific chronic back pain, and exercise programs are the most frequently used treatment method for patients with subacute and chronic back pain ${ }^{5}$. Doing exercise is important to prevent both primary and secondary LBP. The success of an exercise program depends on the adherence of patients to the treatment plan, and on the accurate performance of the exercises. Adherence is defined as the overlapping of an individual's behaviors with the suggestions of medical professionals, and is accepted as the most significant factor in the success of exercise programs; therefore, adherence to exercise programs is crucial ${ }^{6-8)}$. Factors specific to the personal characteristics of the patients, the program, and the interaction between patients and professionals are all known to affect adherence ${ }^{9-13)}$.

The routine practice at our clinic has been to prescribe patient-specific, modified, home-based exercise programs based on the patient's LBP diagnosis, then subsequently invite the patient back for a control evaluation after four weeks. The present study aimed to assess the effectiveness of our practice, and to determine three goals based on the aforementioned information: 1) to examine the rates of patients with chronic, non-specific back pain in order to prescribe home-based exercise

Corresponding author. Sevgi Özdinç (E-mail: sevgiozdinc@yahoo.com)

(C2016 The Society of Physical Therapy Science. Published by IPEC Inc.

This is an open-access article distributed under the terms of the Creative Commons Attribution Non-Commercial No Derivatives (by-nc-nd) License $<$ http://creativecommons.org/licenses/by-nc-nd/4.0/>. 
programs based on a representative population sample; 2) to determine whether the exercises are accurately performed in an unsupervised environment; and 3) to examine the relationships between the adherence to a home-based exercise program and pain level, flexibility, endurance, and disability.

\section{SUBJECTS AND METHODS}

The present study subjects were 49 volunteer patients who presented at the Trakya University Faculty of Medicine, Physical Threapy and Rehabilitation Department. Twenty-eight of 49 patients (57.14\%) did not return for the control evaluation. The sociodemographic features of the 21 volunteer patients who did return for the control evaluation are presented in Table 1. These volunteer patients had had LBP complaints for three months or more, and were referred from polyclinics for a homebased exercise program. The exclusion criteria of our study were: previous surgical operations; mental problems that would have prevented the patient from understanding the exercises; difficulties in performing the exercises due to acute pain; and the inability to return for control evaluation after four weeks. Permission for this study was given by the Trakya University Medicine Faculty Ethic Committee (TÜTFEK-2007/049).

After giving all patients the information and obtaining their consent to participation in the research, demographic and complaint information were collected through a survey based on the statements of the patients. The Oswestry and Rolland Morris scales were used to disability levels, the Visual Analog Scale (VAS) was used to evaluate pain levels, the sit and reach test was used to measure flexibility, and the sit and tuck test was used for endurance.

For the sit and reach test, the patients were asked to touch their toes in an extended sitting position, without bending their knees, while the ankles were in a neutral position. In the event that they were unable to touch their toes, the distance between the middle finger and toes was measured and a negative score was recorded. The score was recorded as zero if the patients just barely touched their toes. If the patient was able to reach further than their toes, the distance was measured and a positive score was recorded ${ }^{14}$.

The sit and tuck test measures the endurance of the rectus abdominus muscle. For this test, the patients were asked to lie down on their backs with their knees bent, and asked to touch their knees with the hands while doing crunches for 30 seconds. Each completed crunch was counted and recorded. The patients whose pain level prevented completion of the test were excluded from the study ${ }^{14)}$. Each exercise suggested for the home program was described and performed. An individualized exercise prescription in the form of a brochure, which included figures and explanations, was given to each participant. In addition, patients were provided with a telephone number to call if they had questions, and a chart on which they were asked to mark each of their prescribed twice-daily sessions. The participants were asked to return to the clinic for control evaluations after four weeks, and were asked to re-perform their exercises. They were given one point for each accurately performed exercise. The maximum possible score was five. Disability, endurance and flexibility were re-evaluated. Statistical analysis was performed using the SPSS 21.0 statistical program. After examining the normality of the data distribution with the one-sample Kolmogorov-Smirnov test, Wilcoxon's two-sample paired test was used for in-group comparisons, since the results of the normality test indicated the data were not normally distributed. Spearman's rho was calculated to determine the relationships among the variables. Arithmetic mean \pm standard deviations of the data are presented in Tables. Significance was accepted for values of $p<0.05$ in all analyses.

Table 1. Subjects' characteristics $(\mathrm{N}=21)$

\begin{tabular}{lc}
\hline Variables & Means \pm Standard deviation \\
\hline Age $(\mathrm{yrs})$ & $43.2 \pm 10.9$ \\
Height $(\mathrm{cm})$ & $164.5 \pm 0.1$ \\
Weight $(\mathrm{kg})$ & $74.8 \pm 13.2$ \\
Body mass index $\left(\mathrm{kg} / \mathrm{m}^{2}\right)$ & $27.7 \pm 4.9$ \\
Complaint duration (months) & $76.5 \pm 65.1$ \\
Gender & Distribution $\%$ \\
Female & 71.4 \\
Male & 28.6 \\
Marital status & \\
Married & 87.5 \\
Single & 12.5 \\
Education & \\
Elementary school & 47.1 \\
High school & 11.8 \\
University & 41.2 \\
\hline
\end{tabular}




\section{RESULTS}

The adherence rate of the home-based exercise program was found to be $54.10 \pm 26.01 \%$ (minimum: $6.6 \%$, maximum: $97 \%$ ), and the accurately performed exercise program score (Accuracy Score) was found to have a value of 2.71 \pm 1.91 . Table 2 presents the statistical significance of improvements in the values of all parameters evaluated in the first evaluation, and again in the control evaluation after four weeks. No correlations were found between the parameters, and the adherence to exercise and accuracy score.

\section{DISCUSSION}

Home-based exercise programs are a practical, economic, and long-term effective treatment method for chronic back pain, and are as effective as standard physical treatment methods, indeed even more effective in the long term ${ }^{12,15-18)}$. The effects of exercise are a decrease in kinesiophobia, the prevention of recurrence, and a decrease in disability. These effects explain the long-term effectiveness of home-based exercise programs ${ }^{7}$. However, adherence is the most critical factor of their effectiveness. Adherence is reported to be approximately $30-50 \%$ for clinically based exercises ${ }^{3)}$. The literature reports adherence as varying between $35-84 \%$ for home-based programs ${ }^{8,9)}$. A Turkish study examined the adherence to home-based exercise programs of musculoskeletal patient groups, and reported an adherence rate of $39 \%{ }^{13)}$. Another study reported regular participation as $35 \%$, and stated that $22 \%$ of participants never adhered to the program ${ }^{9}$. The adherence to home-based exercises by patients with low back pain is reported to be inadequate ${ }^{3,9}$. Not adhering to the exercise prescription affects not only the effectiveness, period, and cost of treatment, and perhaps the statistical results of clinical studies. There are many factors that influence adherence, including age, motivation, pain level, believing in the benefits of the exercise, observing its benefits, adopting a healthful course of action, insufficient time to complete the exercise, social differences, supervision, follow-ups, the communication of the physiotherapist with the patient, the dosage of the program, and the features of exercise prescription $^{13,18,19)}$. The level of complexity of exercises is also a factor which may affect adherence by geriatric individuals ${ }^{9)}$. A recent study also suggested that the number of exercises is an important predictor of adherence ${ }^{20}$. In addition, the factors affecting adherence are different between the periods when pain and inabilities increase, and the extended period when they decrease. It is reported that these factors are similar in periods of pain, but insufficient social support and the patient's physical environment become prominent in an extended period of decreasing pain ${ }^{20)}$. An inverse relationship is reported between the initial pain and adherence. There is strong evidencing link depression, anxiety, self-sufficiency, low social support, and initial low physical activity level to low rates of adherence. Increases in pain during exercises is also a strong obstacle to adherence $^{11)}$. The literature shows there is medium level evidence regarding the fact that living in an environment with high health service standards, and participating in motivational and behavioral change strategies can increase adherence ${ }^{3}$.

The primary way to increase adherence in musculoskeletal patients is to test the effectiveness of exercise at intervals, and measure the adherence to the exercise. Exercises should be prescribed in agreed doses due to the multi-dimensional nature of adherence to exercise. There is no golden standard by which to measure adherence to exercise, although strengthening and flexibility parameters are reported to be the most appropriate methods for measuring adherence ${ }^{5,10)}$. The present study found there were statistically significant improvements in pain, abdominal muscle endurance, flexibility, and disability after home-based exercises were prescribed. However, no significant relationship was found between the outcome measures and the adherence to the exercise program and the accuracy score.

The present study had some limitations. The patients were receiving medical treatment in addition to performing the at-home program. Therefore, we are of the opinion that medical treatment and temporary biological recovery enhanced our results. Additionally, four weeks without supervision or consultation was a long time, and we conclude that this period should be shortened in our routine practice. Home programs should be followed-up, and patients should receive consultations after shorter periods in order to increase adherence.

Table 2. Comparison of the outcome measures between pre- and post-intervention

\begin{tabular}{lcc}
\hline Variables & $\begin{array}{c}\text { First evaluation } \\
\text { Mean } \pm \text { SD }\end{array}$ & $\begin{array}{c}\text { Control evaluation } \\
\text { Mean } \pm \text { SD }\end{array}$ \\
\hline VAS (0-10 point) & $4.84 \pm 4.50$ & $3.26 \pm 3.0^{*}$ \\
Flexibility (cm) & $-4.47 \pm-8.99$ & $-1.88 \pm 7.07^{*}$ \\
Endurance (repetitions) & $8.04 \pm 2.57$ & $10.02 \pm 2.41^{*}$ \\
Oswestry score & $0.38 \pm 0.16$ & $0.26 \pm 0.14^{*}$ \\
Rolland Morris score & $8.09 \pm 6.63$ & $6.28 \pm 5.57^{*}$ \\
\hline
\end{tabular}

*Significant difference, $\mathrm{p}<0.05$ 
In conclusion, the factors affecting adherence to exercise have multi-dimensional features. Even though factors specific to individuals are prominent, social differences can be effective predictors of adherence. Clinicians must estimate an adherence level when recommending home-based exercises, and should make necessary changes to exercise programs in order to increase adherence. Interestingly, even though each exercise was described to the patient by visually performing it, and a descriptive brochure was given to each participant, the exercises were still performed inaccurately. This point should be carefully considered when recommending home programs.

\section{REFERENCES}

1) Karunanayake AL, Pathmeswaran A, Kasturiratne A, et al.: Risk factors for chronic low back pain in a sample of suburban Sri Lankan adult males. Int J Rheum Dis, 2013, 16: 203-210. [Medline] [CrossRef]

2) Metgud DC, Khatri S, Mokashi MG, et al.: An ergonomic study of women workers in a woolen textile factory for identification of health-related problems. Indian J Occup Environ Med, 2008, 12: 14-19. [Medline] [CrossRef]

3) Beinart NA, Goodchild CE, Weinman JA, et al.: Individual and intervention-related factors associated with adherence to home exercise in chronic low back pain: a systematic review. Spine J, 2013, 13: 1940-1950. [Medline] [CrossRef]

4) Alp A, Mengi G, Avsaroglu HA, et al.: Efficiacy of core-stabilization exercise and its comparison with home-based conventional exercise in low back pain patients. Turk J Phys Med Rehab, 2014, 60: 36-42.

5) Holden MA, Haywood KL, Potia TA, et al.: Recommendations for exercise adherence measures in musculoskeletal settings: a systematic review and consensus meeting (protocol). Syst Rev, 2014, 3: 10. [Medline] [CrossRef]

6) Henchoz Y, Kai-Lik So A: Exercise and nonspecific low back pain: a literature review. Joint Bone Spine, 2008, 75: 533-539. [Medline] [CrossRef]

7) Mannion AF, Helbling D, Pulkovski N, et al.: Spinal segmental stabilisation exercises for chronic low back pain: programme adherence and its influence on clinical outcome. Eur Spine J, 2009, 18: 1881-1891. [Medline] [CrossRef]

8) Nava-Bringas TI, Roeniger-Desatnik A, Arellano-Hernández A, et al.: [Adherence to a stability exercise program in patients with chronic low back pain]. Cir Cir, 2016, S0009-7411(15)00274-1. [Medline]

9) Yildirim Y, Soyunov S: Relationship between learning strategies of patients and proper perception of the home exercise program with non-specific low back pain. J Back Musculoskeletal Rehabil, 2010, 23: 137-142. [Medline]

10) Hügli AS, Ernst MJ, Kool J, et al.: Adherence to home exercises in non-specific low back pain. A randomised controlled pilot trial. J Bodyw Mov Ther, 2015, 19: 177-185. [Medline] [CrossRef]

11) Kuukkanen T, Mälkiä E, Kautiainen H, et al.: Effectiveness of a home exercise programme in low back pain: a randomized five-year follow-up study. Physiother Res Int, 2007, 12: 213-224. [Medline] [CrossRef]

12) Ben Salah Frih Z, Fendri Y, Jellad A, et al.: Efficacy and treatment compliance of a home-based rehabilitation programme for chronic low back pain: a randomized, controlled study. Ann Phys Rehabil Med, 2009, 52: 485-496. [Medline] [CrossRef]

13) Chan D, Can F: Patients' adherence/compliance to physical therapy home exercises. Turk J Physiother Rehabil, 2010, 21: 132-139.

14) Özdinc S: Physical Fitness And Postural Characteristics of Choronic Low Back Pain Patients. A Current Perspective on Health Sciences. Editorial: Aysegul Kaptanoglu. Rotipo, 2014, pp 356-363.

15) McCaskey MA, Schuster-Amft C, Wirth B, et al.: Effects of proprioceptive exercises on pain and function in chronic neck- and low back pain rehabilitation: a systematic literature review. BMC Musculoskelet Disord, 2014, 15: 382. [Medline] [CrossRef]

16) Garcia AN, Costa LC, da Silva TM, et al.: Effectiveness of back school versus McKenzie exercises in patients with chronic nonspecific low back pain: a randomized controlled trial. Phys Ther, 2013, 93: 729-747. [Medline] [CrossRef]

17) Nava-Bringas TI, Hernández-López M, Ramírez-Mora I, et al.: Effects of a stabilization exercise program in functionality and pain in patients with degenerative spondylolisthesis. J Back Musculoskeletal Rehabil, 2014, 27: 41-46. [Medline]

18) Escolar-Reina P, Medina-Mirapeix F, Gascón-Cánovas JJ, et al.: How do care-provider and home exercise program characteristics affect patient adherence in chronic neck and back pain: a qualitative study. BMC Health Serv Res, 2010, 10: 60. [Medline] [CrossRef]

19) Medina-Mirapeix F, Escolar-Reina P, Gascón-Cánovas JJ, et al.: Personal characteristics influencing patients' adherence to home exercise during chronic pain: a qualitative study. J Rehabil Med, 2009, 41: 347-352. [Medline] [CrossRef]

20) Eckard T, Lopez J, Kaus A, et al.: Home exercise program compliance of service members in the deployed environment: an observational cohort study. Mil Med, 2015, 180: 186-191. [Medline] [CrossRef] 